\title{
CORRELACIONES FENOTÍPICAS ENTRE CARACTERÍSTICAS PRODUCTIVAS EN ALPACAS HUACAYA
}

\author{
Phenotypic Correlations Between Production Characteristics in the \\ Huacaya Alpaca
}

\begin{abstract}
Alfonso Cordero F. 1,2,3, José Contreras P. ${ }^{2}$, Paúl Mayhua M. ${ }^{2}$, Melanio Jurado E. ${ }^{2}$, Manuel Castrejón V. ${ }^{2}$
\end{abstract}

\section{Resumen}

\begin{abstract}
El presente estudio tuvo como objetivo determinar correlaciones fenotípicas entre peso corporal de la alpaca a la esquila (PCE), peso de vellón sucio (PVS), peso de vellón limpio (PVL), rendimiento de la fibra al lavado (RFL), diámetro medio de las fibras (DMF), longitud de fibra (LF), número de rizos (NR), incidencia de fibras meduladas (IM), peso corporal de la alpaca al parto (PCP) y peso de la cría al nacimiento (PCC). Se trabajó con 185 alpacas Huacaya, blancas, de 1 a 7 años de edad, del Centro Experimental de Camélidos Sudamericanos Lachocc de la Universidad Nacional de Huancavelica, durante los años 2007 y 2008. Las correlaciones fenotípicas fueron: PVS-PVL = 0.99, PVS-LF = $0.55, \mathrm{PVS}-\mathrm{DMF}=0.52, \mathrm{PVS}-\mathrm{PCE}=0.41, \mathrm{PVL}-\mathrm{LF}=0.57, \mathrm{PVL}-\mathrm{DMF}=0.54, \mathrm{PVL}-\mathrm{PCE}=0.43$ $(\mathrm{p}<0.01)$. El peso de vellón no presentó antagonismo con las principales características relacionadas a la producción de fibra, y por la alta interrelación entre el peso de vellón sucio y el diámetro de fibra se puede usar como criterio de selección en un programa de mejoramiento genético que esté orientado a la producción de fibra.
\end{abstract}

Palabras clave: alpaca, Huacaya, interrelaciones productivas

\section{Abstract}

The aim of the present study was to determine phenotypic correlations between body weight of the alpaca at shearing (PCE), greasy fleece weight (PVS), clean fleece weight (PVL), performance of the fibre Andalusia wash (RFL), mean diameter of the fibre (DMF), fibre length (LF), number of loops (NR), incidence of spinal cord fibres (IM), body weight at parturition (PCP) and body weight of calf at birth (PCC). The study considered 185 white Huacaya alpacas, 1 to 7 years old, from the Lachocc Experimental Center of South American Camelids, Universidad Nacional de Huancavelica, in 2007 and 2008. The phenotypic correlations were: $\mathrm{PVS}-\mathrm{PVL}=0.99, \mathrm{PVS}-\mathrm{LF}=0.55, \mathrm{PVS}-\mathrm{DMF}=0.52$, PVS-PCE $=0.41$, PVL-LF $=0.57$, PVL-DMF $=0.54$, PVL-PCE $=0.43(\mathrm{p}<0.01)$. Fleece weight does not show any interference with main characteristics related to fibre production, and

\footnotetext{
${ }^{1}$ Estudio financiado por los recursos del FOCAM

${ }^{2}$ Departamento Académico de Zootecnia, Facultad de Ciencias de Ingeniería, Universidad Nacional de Huancavelica

${ }^{3}$ E-mail: alfonsogcf@hotmail.com
} 
due to the high interrelation between dirty fleece weight and diameter of fibre, it can be used as a valid criterion for selection in a genetic improvement programme aimed at fibre production.

Key words: alpaca, Huacaya, production inter relationships

\section{INTRODUCCIÓN}

Las alpacas constituyen un importante recurso genético animal para los habitantes de las regiones alto-andinas del Perú, Bolivia, Argentina y Chile, pues son fuentes generadoras de carne, pieles, trabajo y, sobre todo, de fibra de gran valor económico. Además, permiten el aprovechamiento de la tierra en zonas geográficas donde solo puede explotarse animales adaptados al medio.

Se reconocen dos razas de alpacas, Huacaya y Suri. En el Perú, la Huacaya, con una población de 2.3 millones de cabezas (Flores, 2002), se caracteriza por ser corpulenta, de mayor fortaleza y tener el vellón de apariencia esponjosa, en relación a la Suri, que muestra formas angulosas y textura más fina con vellón ligeramente más fino, pesado, brillante y suave.

Existen diversos factores que influyen en la producción de fibra, muchos de ellos interrelacionados. La evaluación de los animales con respecto a estas características se realiza directamente a través del fenotipo del individuo, o se estima a través de la genealogía o de su descendencia. Al seleccionar animales por una determinada característica, se debe considerar que, indirectamente, otras variables estarán siendo afectadas. Las correlaciones fenotípicas permiten predecir cambios de una característica en el rebaño actual, cuando se selecciona animales por una u otra característica (Lush, 1965; Van Vleck et al., 1987; Lopes et al., 2005). El valor absoluto de la correlación indica si la asociación es alta o baja, facilitando la selección cuando las correlaciones son del mismo signo, o debiendo ponderarlas económicamente cuando son de signo contrario.
En la zona alto andina del país, la crianza de la alpaca está orientada a la producción de fibra (Wuliji et al., 2000; Caballero y Flores, 2004), siendo el principal criterio de selección el peso de vellón. No obstante, no se consideran otros parámetros como el diámetro de fibra que es la característica más importante en la clasificación de la fibra; el mismo que determina, generalmente, el precio de la fibra en el mercado (Gonzales et al., 2008).

Se dispone de poca información de varias características productivas, incluyendo la medulación de las fibras, el peso corporal de la alpaca al momento del parto y el peso de la cría al nacimiento. Se encontraron coeficientes de correlación de 0.99 entre el peso de vellón sucio y limpio en crías y tuis de la raza Huacaya en Nueva Zelanda (Wuliji et al., 2000); en tanto que en ovinos Polwarth brasileros se ha reportado un valor de 0.94 para estas características (Osório et al., 1981). Asimismo, en la zona de Cajamarca, Perú, se encontró valores de 0.50 y 0.71 entre el peso de vellón sucio y longitud de fibra, dependiendo de la zona geográfica (Raunelli y Coronado, 2006).

El diámetro de las fibras es la característica más importante, en relación a su calidad, desde el punto de vista de su transformación en artesanías o comercialización por empresas textiles locales o extranjeras, de modo que las fibras de menor diámetro serán utilizadas en la confección de prendas más finas. Resultados obtenidos por Wuliji et al. (2000), Raunelli y Coronado (2006) y Quispe et al. (2008) revelan una correlación positiva entre peso de vellón sucio y diámetro de fibras, que varía entre 0.12 a 0.47 . En ovinos, este coeficiente de correlación fluctúa entre 0.13 y 0.40 (Mullaney et al., 1970). Por otro 
lado, la asociación fenotípica entre el peso corporal y el peso de vellón sucio en la alpaca se encuentra entre 0.25 y 0.58 (Bustinza, 1991; Chávez, 1991). El objetivo del presente estudio fue identificar relaciones funcionales entre las características productivas de alpacas hembras adultas, con mayor énfasis en el peso de vellón sucio y sus principales componentes.

\section{Materiales y Métodos}

Se utilizaron 185 alpacas Huacaya, hembras, blancas, del Centro Experimental de Camélidos Sudamericanos Lachocc, de la Universidad Nacional de Huancavelica, situado cerca de la ciudad de Huancavelica, entre 4100 y 4900 msnm. El estudio se llevó a cabo entre noviembre de 2007 y abril de 2008. La esquila de los animales se realizaba anualmente y el pastoreo se realizaba entre las 08 y 16 horas en pastizales no sometidos a riego, y constituidos principalmente por Alchemilla pinnata, Festuca dolichophylla, Poa alquigluma, Calamagrostis curvula, Plantago rigida y Stipa obtusa.

Los animales estuvieron bajo condiciones similares de manejo y sanidad. Se tomó en consideración el estado reproductivo (vacía, gestante, lactante), y el peso corporal al parto de la madre (PCP) y de la cría al nacimiento (PCC). La edad (en años) se obtuvo de los registros individuales de los animales.

Se tomó una muestra de fibra de aproximadamente $30 \mathrm{~g}$ de la región del costillar izquierdo de cada animal, previo a la esquila mecánica (Aylan-Parker y McGregor, 2002). Luego de la esquila se registró el peso corporal (PCE) con una balanza de $100 \mathrm{~kg}$ de capacidad, y los pesos del vellón sucio (PVS) y de bragas (PB) con una balanza electrónica de una sensibilidad de $5 \mathrm{~g}$.

Las muestras se trasladaron al laboratorio del Programa Ovinos y Camélidos de la Universidad Nacional Agraria La Molina, en Lima; donde se determinó el peso del vellón limpio (PVL), rendimiento de fibra al lavado (RFL), diámetro medio de fibra (DMF), longitud de fibra (LF), número de rizos (NR), e incidencia de medulación (IM).

En la determinación del diámetro medio de fibras se usó las recomendaciones de la norma de la Sociedad Americana de Pruebas y Materiales (ASTM, 1998), Designación D 6466-99 (Reapproved 2005). Para el rendimiento al lavado, se siguió las correlaciones de la Designación 6: 4-005-75 de la Comisión Panamericana de Normas Técnicas (COPANT, 1975). Las muestras fueron sometidas a un proceso de lavado en el Leviatán. El peso de vellón limpio fue determinado tomando como base el rendimiento de la fibra al lavado y el peso de vellón sucio $[\mathrm{PVL}=(\mathrm{PVS} \times \mathrm{RFL}) / 100]$. Para la determinación de la longitud de fibra se utilizó el método de la ASTM (ASTM, 1999), Designación D 1234-85, Reapproved 2001. Para el número de rizos se usó un tablero de fondo negro, lupa, pinzas y una regla graduada en centímetros para efectuar el conteo de rizos en forma visual. La medulación de las fibras fue determinada en concordancia con la norma de la ASTM, Designación 2968-89 (ASTM, 1991). Los datos porcentuales de las características en estudio fueron transformados a raíz cuadrada del porcentaje de arco seno utilizando Microsoft Excel®.

Las correlaciones fenotípicas de las características en estudio fueron calculadas después del ajuste de los datos por edad (E) y condición reproductiva de la alpaca (C) en base al procedimiento CORR del programa estadístico SAS (SAS, 1999), a través del modelo estadístico:

$\mathrm{Y}_{\mathrm{ijk}}=\mu+\mathrm{E}_{\mathrm{i}}+\mathrm{C}_{\mathrm{j}}+\mathrm{e}_{\mathrm{ikj}}$, donde:

$\mathrm{Y}_{\mathrm{ijk}}=\mathrm{PCE}(\mathrm{o}$ PVS, PB, PVL, RFL, DMF, LF, NR, IM, PCP o PCC) en $\mathrm{k}$ observaciones de la edad i y condición reproductiva j;

$\mu=$ media general, común a todas las observaciones;

$E_{i}=$ efecto de la edad $y$, variando de $1 \mathrm{a} 7$; 
$\mathrm{C}_{\mathrm{j}}=$ efecto de la condición reproductiva $\mathrm{j}$, variando de 1 a 3 .

$\mathrm{e}_{\mathrm{ikj}}=$ error aleatorio asociado a la observación $\mathrm{Y}_{\mathrm{ijk}}$, tal que $\sim \mathrm{N}\left(\mathrm{o} ; \tilde{\mathrm{o}}^{2}\right)$

Para el cálculo del error estándar de cada coeficiente de correlación se consideró la fórmula utilizada por Fisher (1954), citado por Cordero (1979). Se tuvo 185 determinaciones por característica, excepto para el PCP y PCC que consistió en 25 determinaciones.

\section{Resultados y Discusión}

En el Cuadro 1, se muestra la distribución de las alpacas, de acuerdo a la edad y la condición reproductiva y en el Cuadro 2 se indican los resultados de las mediciones de las características productivas de las alpacas. En general, puede considerarse como baja la variabilidad del PCP, DMF, PCC y PCE, y alta la variación del PVS, PVL y LF.

Cuadro 1. Distribución de las alpacas por edad y condición reproductiva

\begin{tabular}{ccccc}
\hline \multirow{2}{*}{ Edad (años) } & \multicolumn{3}{c}{ Condición reproductiva } & \multirow{2}{*}{ Total } \\
\cline { 2 - 4 } & Vacías & Gestantes & Lactantes & \\
\hline 1 & 46 & - & - & 46 \\
2 & 11 & 2 & 6 & 19 \\
3 & 13 & 15 & - & 28 \\
4 & 9 & 9 & 3 & 21 \\
5 & 4 & 10 & 3 & 17 \\
6 & 6 & 4 & 4 & 14 \\
7 & 16 & 15 & 9 & 40 \\
\hline Total & 105 & 55 & 25 & 185 \\
\hline
\end{tabular}

Cuadro 2. Promedios y coeficientes de variabilidad de características productivas de alpacas Huacaya

\begin{tabular}{lccc}
\hline Características & Promedio & $\begin{array}{c}\text { Desviación } \\
\text { estándar }\end{array}$ & $\begin{array}{c}\text { Coeficiente de } \\
\text { variación (\%) }\end{array}$ \\
\hline $\begin{array}{l}\text { Peso corporal a la esquila } \\
\text { (PCE, kg) }\end{array}$ & 56.62 & 12.63 & 0.22 \\
$\begin{array}{l}\text { Peso de vellón sucio } \\
\text { (PVS, kg) }\end{array}$ & 2.52 & 0.98 & 0.38 \\
$\begin{array}{l}\text { Peso vellón limpio } \\
\text { (PVL, kg) }\end{array}$ & 2.28 & 0.88 & 0.39 \\
$\begin{array}{l}\text { Diámetro medio de la fibra } \\
\text { (DMF, } \mu \text { m) }\end{array}$ & 23.42 & 4.14 & 0.18 \\
$\begin{array}{l}\text { Longitud de la fibra } \\
\text { (LF, plg) }\end{array}$ & 4.15 & 1.33 & 0.32 \\
$\begin{array}{l}\text { Peso vivo al parto } \\
\text { (PCP, kg) }\end{array}$ & 65.12 & 6.37 & 0.09 \\
$\begin{array}{l}\text { Peso cría al nacer } \\
\text { (PCC, kg) }\end{array}$ & 7.72 & 1.52 & 0.20 \\
\hline
\end{tabular}


Cuadro 3. Correlaciones fenotípicas ${ }^{1}$ de características productivas de alpacas Huacaya

\begin{tabular}{|c|c|c|c|c|c|c|c|c|c|c|}
\hline & PCE & PVS & PB & PVL & RFL & DMF & LF & NR & IM & PCP \\
\hline PVS & $\begin{array}{l}0.41 * * * \\
\pm 0.06\end{array}$ & & & & & & & & & \\
\hline PB & $\begin{array}{l}0.20 * * \\
\pm 0.07\end{array}$ & $\begin{array}{l}0.28 * * \\
\pm 0.07\end{array}$ & & & & & & & & \\
\hline PVL & $\begin{array}{l}0.43 * * * \\
\pm 0.06\end{array}$ & $\begin{array}{l}0.99 * * * \\
\pm 0.00\end{array}$ & $\begin{array}{l}0.25 * * \\
\pm 0.07\end{array}$ & & & & & & & \\
\hline RFL & $\begin{array}{l}0.21 * * \\
\pm 0.07\end{array}$ & $\begin{array}{l}0.01 \\
\pm 0.07\end{array}$ & $\begin{array}{l}-0.16^{*} \\
\pm 0.07\end{array}$ & $\begin{array}{l}0.11 * * \\
\pm 0.07\end{array}$ & & & & & & \\
\hline DMF & $\begin{array}{l}0.59 * * * \\
\pm 0.05\end{array}$ & $\begin{array}{l}0.52 * * * \\
\pm 0.05\end{array}$ & $\begin{array}{l}0.25 * * \\
\pm 0.08\end{array}$ & $\begin{array}{l}0.54 * * * \\
\pm 0.05\end{array}$ & $\begin{array}{l}0.29 * * * \\
\pm 0.07\end{array}$ & & & & & \\
\hline $\mathrm{LF}$ & $\begin{array}{l}0.02 \\
\pm 0.07\end{array}$ & $\begin{array}{l}0.55 * * * \\
\pm 0.05\end{array}$ & $\begin{array}{l}-.15^{*} \\
\pm 0.07\end{array}$ & $\begin{array}{l}0.57 * * * \\
\pm 0.05\end{array}$ & $\begin{array}{l}0.14^{*} \\
\pm 0.07\end{array}$ & $\begin{array}{l}0.24 * * \\
\pm 0.07\end{array}$ & & & & \\
\hline NR & $\begin{array}{l}-.35 * * * \\
\pm 0.07\end{array}$ & $\begin{array}{l}-.17 * \\
\pm 0.07\end{array}$ & $\begin{array}{l}-.27 * * * \\
\pm 0.07\end{array}$ & $\begin{array}{l}-.17 * \\
\pm 0.07\end{array}$ & $\begin{array}{l}-.08 \\
\pm 0.07\end{array}$ & $\begin{array}{l}-.49 * * \\
\pm 0.06\end{array}$ & $\begin{array}{l}0.12 \\
\pm 0.07\end{array}$ & & & \\
\hline $\mathrm{IM}$ & $\begin{array}{l}0.33 * * * \\
\pm 0.07\end{array}$ & $\begin{array}{l}0.33 * * * \\
\pm 0.07\end{array}$ & $\begin{array}{l}0.16^{*} \\
\pm 0.07\end{array}$ & $\begin{array}{l}0.34 * * * \\
\pm 0.07\end{array}$ & $\begin{array}{l}0.12 \\
\pm 0.07\end{array}$ & $\begin{array}{l}0.62 * * * \\
\pm 0.06\end{array}$ & $\begin{array}{l}0.20 * * \\
\pm 0.07\end{array}$ & $\begin{array}{l}-.30 * * * \\
\pm 0.07\end{array}$ & & \\
\hline PCP & $\begin{array}{l}0.90 * * \\
\pm 0.04\end{array}$ & $\begin{array}{l}0.73 * * \\
\pm 0.09\end{array}$ & $\begin{array}{l}0.39 * \\
\pm 0.17\end{array}$ & $\begin{array}{l}0.73 * * \\
\pm 0.09\end{array}$ & $\begin{array}{l}-.27 \\
\pm 0.19\end{array}$ & $\begin{array}{l}0.39^{*} \\
\pm 0.17\end{array}$ & $\begin{array}{l}0.48 * \\
\pm 0.15\end{array}$ & $\begin{array}{l}0.10 \\
\pm 0.20\end{array}$ & $\begin{array}{l}0.22 \\
\pm 0.19\end{array}$ & \\
\hline PCC & $\begin{array}{l}0.53 * * \\
\pm 0.14\end{array}$ & $\begin{array}{l}0.67 * * \\
\pm 0.11\end{array}$ & $\begin{array}{l}0.40^{*} \\
\pm 0.17\end{array}$ & $\begin{array}{l}0.69 * * \\
\pm 0.10\end{array}$ & $\begin{array}{l}-.30 \\
\pm 0.18\end{array}$ & $\begin{array}{l}0.30 \\
\pm 0.18\end{array}$ & $\begin{array}{l}0.65 * * \\
\pm 0.12\end{array}$ & $\begin{array}{l}0.30 \\
\pm 0.18\end{array}$ & $\begin{array}{l}0.31 \\
\pm 0.18\end{array}$ & $\begin{array}{l}0.62 * * \\
\pm 0.12\end{array}$ \\
\hline
\end{tabular}

${ }^{* * *}: p<0.001 ;{ }^{* *}: p<0.01 ;{ }^{*}: p<0.05$

${ }^{1}$ PVS: Peso de vellón sucio; PB: Peso de bragas; PVL: Peso vellón limpio; RFL: Rendimiento de fibra lavada; DMF: Diámetro medio de la fibra; LF: Longitud de la fibra; NR: número de rizos; IM: Índice de modulación; PCE: Peso corporal a la esquila; PCP: Peso corporal al parto; PCC: Peso de la cría al nacimiento

Las correlaciones fenotípicas entre las variables en estudio se encuentran en el Cuadro 3. Se observa una correlación positiva (r $=0.99)$ muy alta entre el peso de vellón sucio y peso de vellón limpio $(\mathrm{p}<0.001)$, siendo estas dos variables de gran importancia en programas de selección con fines de mejoras en la producción de fibra. Así, cuando fuera inviable o antieconómico determinar el peso de vellón limpio, se puede usar el peso de vellón sucio como criterio principal en un programa de selección (Wuliji et al., 2000).

Las correlaciones positivas altas obtenidas entre el peso de vellón (sucio y limpio) y la longitud de fibra $(r=0.55$ y $r=0.57$, $\mathrm{p}<0.001)$ permiten una selección indirecta de alpacas con mayor longitud de fibra al seleccionar por peso de vellón sucio. Los valores de estas correlaciones fueron similares a valores encontrados por Wuliji et al. (2000). Correlaciones positivas similares se encontró entre peso de vellón (sucio y limpio) y diámetro de fibras $(\mathrm{r}=0.52$ y $\mathrm{r}=0.54$, $\mathrm{p}<0.001$ ), indicando que entre los animales de mayor peso de vellón se encuentran alpacas con mayor diámetro de fibras. Otros reportes muestran correlaciones ligeramente inferiores para esta característica (Wuliji et al., 2000; Raunelli y Coronado, 2006).

Otra variable de interés es el peso corporal a la esquila, pues esta característica está asociada a la tasa reproductiva. Se encon- 
traron relaciones fenotípicas positivas medianamente altas del peso corporal a la esquila y el peso de vellón sucio $(0.41, \mathrm{p}<0.001)$ y peso de vellón limpio $(0.43, \mathrm{p}<0.001)$ y muy alta asociación entre el peso corporal de la alpaca al parto y peso de vellón sucio $(0.73$, p<0.01). Bustinza (1991) y Chávez (1991) señalan coeficientes de correlación entre el peso corporal a la esquila y peso de vellón sucio entre 0.25 y 0.58 .

La relación fenotípica entre el rendimiento al lavado y el peso de vellón sucio $(\mathrm{r}=$ $0.01)$ y vellón limpio $(\mathrm{r}=0.11)$ fue muy baja, e inferior a otros reportes (Wuliji et al., 2000).

\section{Conclusiones}

? Los coeficientes de correlación fenotípicas en alpacas Huacaya no muestran antagonismo entre el peso de vellón sucio y las principales variables de interés económico.

? Al seleccionar alpacas por peso de vellón, se selecciona indirectamente animales de mayor peso corporal y longitud de fibra.

\section{Literatura Citada}

1. ASTM. 1991. Standard test method for Med and Kemp fiber in wool and other animal fibers by microprojection. In: Annual Book of ASTM, Designation. D 2968-89. Vol 7(01). Philadelphia: ASTM. p 809-811.

2. ASTM. 1998. Anual Book of Standards American Society for Testing and Materials. Editorial Staff. PhiladelphiaUSA.

3. ASTM. 1998. Standard test method for diameter of wool and animal fibers by Sirolan-Laserscan fiber diameter analyser. In: Annual Book of ASTM, Designation: D 6466-99 (Reapproved 2005). Philadelphia: ASTM. p 1-9.
4. ASTM. 1999. Standard test method of sampling and testing stage length of grease wool. In: Annual Book of ASTM Standards, Designation: D 1234-85 (Reapproved 2001). Vol 07(01). Philadelphia: ASTM. p 1-4.

5. Aylan-Parker J, Mcgregor B. 2002. Optimizing sampling techniques and estimating sampling variance of fleece quality attributes in alpacas. Small Ruminant Res 44: 53-64.

6. Bustinza V. 1991. Mejoramiento genético. En: Novoa C, Flores A (eds). Producción de rumiantes menores: alpacas. California, EEUU: Programa de Apoyo a la Investigación Colaborativa en Rumiantes Menores (SR-CRSP)Convenio Universidad de California, Davis-INIAA. p 113-128.

7. Caballero W, Flores A. 2004. La sierra. Primera prioridad para salir del subdesarrollo agrario. Lima: CONCYTEC. 285 p.

8. Chávez C. 1991. Plan para el mejoramiento genético de alpacas. En: Novoa C, Flores A (eds). Producción y rumiantes menores: Alpacas. California, EEUU: Programa de Apoyo a la Investigación Colaborativa en Rumiantes Menores (SR-CRSP)-Convenio Universidad de California, Davis-INIAA. p 129-145.

9. [COPANT] Comisión Panamericana de Normas Técnicas. 1975. Designación 6:4-005-75. Company, Inc. 481. Philadelphia, EEUU: Ed Staff.

10. Cordero A. 1977. Estudios de las características productivas en ganado Holstein de la cuenca lechera de Lima. Tesis de Maestría en Ciencias. Lima: Univ Agraria La Molina.

11. Flores A. 2002. Visión panorámica de la ganadería. En: Caballero W, Flores A, Arroyo O, Alcántara A (eds). Hacia una nueva agricultura, con énfasis en la generación y transferencia de tecnología. Lima: CONCYTEC. p 245-314.

12. Gonzáles H, León-Velarde C, Rosadio $R$, García W, Gavidia C. 2008. Evaluación de un método numérico de medición del diámetro de la fibra de alpaca. Rev Inv Vet, Perú 19: 1-8. 
13. Lopes P, Pieres A, Filho J, Tores R. 2005. Teoria do melhoramento animal. Belo Horizonte, Brasil: FEPMVZ. 118 p.

14. Mullaney P, Brown G, Young, Hyland $P .1970$. Genetic and phenotypic parameter for wool characteristics in fine-wool Merino, Corriedale and Polwarth shep. II. Phenotypic and genetic correlation, heritability and repeatability. Austr J Agric Res 21: 527-540.

15. Osório J, Cardellino R, Ribeiro J, Fernandes J, Guerreiro J, Da Silveira O. 1981. Relação entre características productivas em ovelhas da raça Ideal (Polwarth). Rev Zoot Bras Zootecnia 10(1): 39-49.

16. Quispe P, Alfonso L, Flores A, Guillén H. 2008. Bases para establecer un programa de mejora de alpacas en la región alto andina de Huancavelica-Perú. En: Quispe E, Mueller J, Ruiz J, Alfonso L, Gutiérrez G (eds). Actualidades sobre adaptación, producción, reproducción y mejora genética en camélidos. Huancavelica: Ed Gráfica Industrial. p 93-112.

17. Raunelli S, Coronado L. 2006. Un método de selección aplicable a alpacas. En: II Simposium Internacional de Investigaciones sobre Camélidos Sudamericanos. Arequipa. p 21-35.

18. [SAS] Statistical Analysis System. 1999. SAS user' guide: statistics. Cary: SAS Institute. $965 \mathrm{p}$.

19. Van Vleck LD, Pollack EJ, Oltenacu EAB. 1987. Genetics for the animal sciences. New York: Freeman WH. 391 p.

20. Wuliji T, Davis G, Dodds K, Turner P, Andrews R, Bruce G. 2000. Production performance, repeatability and heritability estimates for live weight, fleece weight and characteristics of alpacas in New Zealand. Small Ruminant Res 37: 189-201. 